\title{
Improving Functionalities in a Multi-agent Architecture for Ocean Monitoring
}

Fernando de la Prieta, Oscar Gil, Carolina Zato, Beatriz Martín, and Alberto García

\begin{abstract}
This paper presents an improved version of a multiagent architecture aimed at providing solutions for monitoring the interaction between the atmosphere and the ocean. The ocean surface and the atmosphere exchange carbon dioxide. This process is can be modeled by a multiagent system with advanced learning and adaption capabilities. The proposed multiagent architecture incorporates CBRagents. The CBR-agents proposed in this paper integrate novel strategies that both monitor the parameters that affect the interaction, and facilitate the creation of models. The system was tested and this paper presents the results obtained.
\end{abstract}

Keywords: Multi-Agent Systems, Intelligent agents, Distributed Computing.

\section{Introduction}

Multi-agent systems are very appropriate for resolving problems in a distributed way [15]. Agents have a set of characteristics, such as autonomy, reasoning, reactivity, social abilities, pro-activity, mobility, organization, etc. which allow them to cover several needs for dynamic environments. Agent and multi-agent systems have been successfully applied to several scenarios, such as education, culture, entertainment, medicine, robotics, etc. [3], [21]. Moreover, the continuous advancement in mobile computing makes it possible to obtain information about the context and also to react physically to it in more innovative ways [15]. Nevertheless, complex systems need higher adaptation, learning and autonomy levels than pure BDI model [1]. This can be achieved by modelling the agents' characteristics [23] to provide them with mechanisms that allow solving complex problems and autonomous learning [8].

One of the factors of greatest concern in climactic behaviour is the quantity of carbon dioxide present in the atmosphere. Carbon dioxide is one of the greenhouse

Fernando de la Prieta $\cdot$ Oscar Gil · Carolina Zato · Beatriz Martín · Alberto García

Facultad de Ciencias

Universidad de Salamanca

Plaza de la Merced s/n, 37008, Salamanca, Spain

e-mail: \{fer, oscar.gil, carol_zato, eureka, alberto_gar\}@usal.es

Y. Demazeau et al. (Eds.): Trends in PAAMS, AISC 71, pp. 555-562.

springerlink.com $\quad$ C Springer-Verlag Berlin Heidelberg 2010 
gases that helps to make the earth's temperature habitable, so long as it is maintained at a certain level [20]. The main system regulating carbon dioxide in the atmosphere has traditionally been thought to be the photosynthesis and respiration of plants. However, teledetection techniques have been able to show that the ocean plays a highly important role in the regulation of carbon quantities, although the full significance of this still needs to be determined $[12,20]$. Current technology allows us to obtain data and make calculations that were unthinkable some time ago. This data provides an insight into the original source of carbon dioxide, the decrease in carbon dioxide, and the causes for both [16], which allows us to make predictions about the behaviour of carbon dioxide in the future.

The aim of the present study is to improve the functionalities of an architecture that makes it possible to construct dynamic systems capable of growing in dimension and adapting their knowledge to environmental changes [3, 8]. The mission of the multiagent system is to globally monitor the interaction between the ocean surface and the atmosphere, facilitating the work of oceanographers. The system is being used in order to evaluate and predict the amount of carbon dioxide $\left(\mathrm{CO}_{2}\right)$ absorbed or expelled by the ocean in the North Atlantic [6]. The CBR-BDI agents [5] presented in the framework of this research incorporate innovative techniques in each of the stages of the CBR cycle. The retrieve phase incorporates a novel strategy based on growing cell structure neural network that provides a set of cases grouped in meshes according to similarity criteria. The reuse phase is composed of a multilayer perceptron neural network and a Jacobean sensitive matrix. The revise phase is carried out by means of a pondered weight technique. Finally, the retain stage updates the growing cell structure neural network.

The next section reviews the environmental problem that motivates the majority of this research. Section three describes the multiagent architecture specifically developed to monitor the air-sea interaction. Section four presents the CBR-BDI agent based system developed. Finally the conclusions and some preliminary results are presented.

\section{Problem Description and Background}

In recent years a great interest has emerged in climactic behaviour and the impact that mankind has had on the climate. One of the most worrying factors is the quantity of $\mathrm{CO}_{2}$ present in the atmosphere. Until only a few years ago, the photosynthesis and breathing processes in plants were considered to be the regulatory system that controls the presence of $\mathrm{CO}_{2}$ in the atmosphere. However, the role of the ocean in the regulation of carbon volume is very significant and so far remains indefinite [19]. Current technology makes it possible to obtain data and estimates that were beyond expectations only a few years ago. The goal of this project is to construct a model that calculates the global air-sea flux of $\mathrm{CO}_{2}$ exchanged between the atmosphere and the surface waters of the ocean. In order to create a new model for the $\mathrm{CO}_{2}$ exchange between the atmosphere and the oceanic surface a number of important parameters must be taken into consideration: sea surface temperature, air temperature, sea surface salinity, atmospheric and hydrostatic pressures, the presence of nutrients and the wind speed vector (module and direction) [20]. 
These parameters can be obtained from oceanographic ships as well as from satellite images. Satellites provide a great amount of daily information and there is a growing need for the ability to automatically process and learn from this source of knowledge. These parameters allow us to calculate the variables that define our models, such as the velocity of gas transfer, solubility, or the differentiation between partial pressures on the atmosphere and sea surface (a case structure is shown in Table 1).

Table 1 Case Attributes

\begin{tabular}{ll}
\hline Case Field & Measurement \\
\hline DATE & Date $(\mathrm{dd} / \mathrm{mm} /$ yyyy) \\
LAT & Latitude (decimal degrees) \\
LONG & Longitude (decimal degrees) \\
SST & Temperature $\left({ }^{\circ} \mathrm{C}\right)$ \\
S & Salinity (unitless) \\
WS & Wind strength $(\mathrm{m} / \mathrm{s})$ \\
WD & Wind direction (unitless) \\
Fluo_calibrated & fluorescence calibrated with chlorophyll \\
SW pCO $\mathrm{CO}_{2}$ & surface partial pressure of $\mathrm{CO}_{2}$ (micro Atmospheres) \\
Air pCO & air partial pressure of $\mathrm{CO}_{2}\left(\mathrm{micro}_{2}\right.$ Atmospheres) \\
Flux of $\mathrm{CO}_{2}$ & $\mathrm{CO}_{2}$ exchange flux (Moles $\left./ \mathrm{m}^{2}\right)$ \\
\hline
\end{tabular}

\section{Multiagent System for Predicting the Ocean Behaviour}

To handle all the potentially useful data to create daily models in a reasonable time and with a reasonable cost, it is necessary to use automated distributed systems capable of incorporating new knowledge. Our proposal consists of a multiagent system whose main characteristic is the use of CBR-BDI agents. The architecture was detailed in previous works $[3,8]$ and is shown in Figure 1.

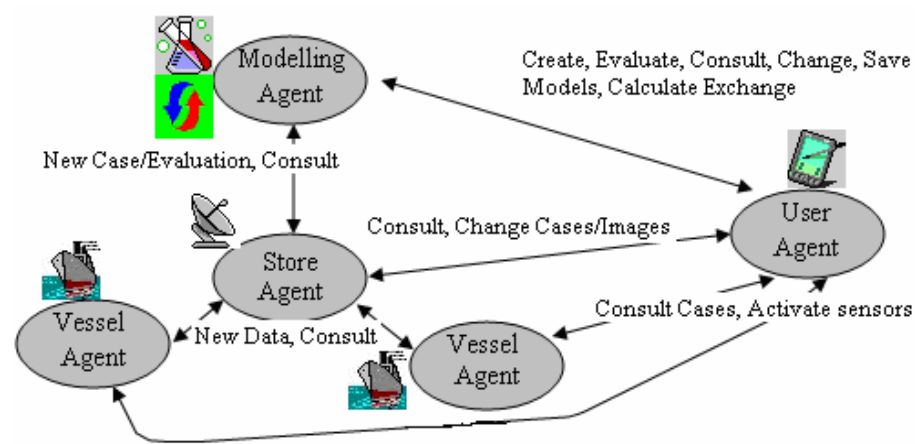

Fig. 1 Diagram of the architecture of our MAS 
Figure 1 illustrates a multiagent system in which is it possible to observe how a Modelling agent with a CBR-BDI architecture is responsible for the creation and evaluation of models in terms of the data received from the Store, Vessel and User agents. This model makes it possible to monitor and predict the carbon dioxide exchange between the ocean surface and the atmosphere. The Store agent processes the images from the satellite and transforms them for use by the system. Each Vessel agent is installed in a ship and collects information in-situ that makes it possible to evaluate the models created by the Modelling agent. The User agent can interact with any of the other agents [22]. Figure 1 shows how the agents interact with each other and with their surroundings [1, 4]. In order to resolve the problem from an oceanographic perspective, the ocean was divided into a series of zones in each of which there is a Modelling Agent, a Store Agent, and various Vessel Agents.

This paper presents an improved version of the Modelling agent [3, 8], incorporating novel strategies in the retrieve, reuse and revise stages of the CBR-agent cycle. These innovative strategies are presented in detail in the following section.

\section{Agents with Advanced Prediction Abilities}

The deliberative agents proposed in the framework of this investigation use the concept of Case-based Reasoning (CBR), a type of reasoning based on the use of past experiences [14], to gain autonomy and improve their problem-solving capabilities. The method proposed in [5] facilitates the incorporation of case-based reasoning systems as a deliberative mechanism within BDI agents, allowing them to learn and adapt themselves, lending them a greater level of autonomy than pure BDI architectures [1]. Accordingly, CBR-agents implemented using case-based reasoning systems can reason autonomously and therefore adapt themselves to environmental changes. The case-based reasoning system is completely integrated within the agents' architecture.

The Modelling agent, a CBR-BDI agent that has two principal functions. The first function is to generate models that are capable of predicting the atmospheric/oceanic interaction in a particular area of the ocean in advance. The second is to permit the use of such models. The reasoning cycle of a CBR system is included among the activities, and is comprised of the retrieval, reuse, revise and retain stages. An additional stage is used to introduce an expert's knowledge. This reasoning cycle must correspond to the sequential execution of some of the agent roles. The Modelling agent carries out roles to generate models such as Jacobean Sensitivity Matrix (JSM), Pondered Weigh Technique (PWT), Revision Simulated Equation (RSE), and other roles that allow it to operate with the calculating models, like Forecast Exchange Rate, Evaluate Model or Consult model. The roles used to carry out the stages of the CBR cycle are described as follows.

The content of the information stored in the memory of cases for each of the cases is described in Table 1 . As can be seen, a case consists of a series of variables that can be represented as a tuple $\mathrm{c}=(\mathrm{d}, \mathrm{l}, \mathrm{o}, \mathrm{t}, \mathrm{s}, \mathrm{w}, \mathrm{r}, \mathrm{f}, \mathrm{p}, \mathrm{a}, \mathrm{M}, \mathrm{e}, \mathrm{x})$, where $\mathrm{d}$ represents the date, 1 the latitude, o the longitude, $t$ the temperature, $s$ the salinity, $w$ the wind strength, wd the wind direction, $f$ the fluorescence calibrated, $s$ the 
surface partial pressure of $\mathrm{CO}_{2}$, a the air partial pressure of $\mathrm{CO}_{2}, \mathrm{M}$ the Multilayer Perceptron (MLP) associated to the case, e the $\mathrm{CO}_{2}$ Flux, and $\mathrm{i}$ the exchange value. The memory of cases is defined as a set of cases and is represented as $\mathrm{C}=\{\mathrm{c}\}$. When a new problem is studied, the system incorporates a new case $\mathrm{c}_{\mathrm{n}+1}$ and a new CBR cycle is executed:

Retrieve phase: The retrieval process identifies those cases in the memory of cases that have the highest level of similarity with the new case $c_{n+1}$. In order to do so, the memory of cases is structured in such a way so as to group together the most similar cases. GCS [13] (Growing Cell Structure) is used at this stage, since GCS does not set the number of neurons, or the degree of connectivity. GCS networks adjust the data by means of a series of disconnected meshes that are obtained during the training stage of the neural network. In this sense, the neural network provides a series of distributed meshes that represent the memory of cases. Each of the cases of the memory of cases is assigned to the nearest mesh, so when a new case is studied, the closest mesh is selected along with the cases associated to the mesh. These are the cases that will be used in the reuse phase. If there exists is a neural network that has been previously trained with the set of retrieved cases, that is, a CBR cycle previously executed with the cases of the selected mesh, then the settings of the neural network are reloaded. When the training process finishes, the result obtained is a set of cases grouped in meshes that are represented as $G=\left\{g_{i} / g_{i} \subseteq C\right\}$, where $g_{i} \cap g_{j}=\phi \forall i \neq j$.

Reuse phase: This phase is carried out by means of a multilayer perceptron [18]. The MLP makes only use of the data recovered in the retrieve phase instead of working with all the data stored in the memory of cases. This fact provides a notable reduction in the time required for the training stage of the MLP, and improves the prediction provided by the neural network since the data are more homogeneous. When the group $g_{i}$ has already executed a Reuse phase and, as a result, it is associated with a previously trained MLP, then it is necessary to calculate the estimate error rate for the cases used by the MLP. If the condition established by Equation (1) is met, the training stage is not carried out.

$$
\frac{\sum_{i=1}^{N}\left|M_{g_{j}}\left(c_{i}\right)-x_{i}\right|}{N \cdot \bar{x}}<\mu
$$

where $\mathrm{N}$ represents the set of cases for the group $\mathrm{g}_{\mathrm{j}}, M_{g_{j}}\left(c_{i}\right)$ is the value estimated by the MLP for the case $c_{i}, x_{i}$ is the exchange value, and $\mu$ is the threshold that identifies the limit considered as valid. Otherwise, when a MLP does not previously exist, it is necessary to execute the training phase before making predictions. To carry out the training phase of the MLP, it is necessary to readjust the data in such a way that all the data are normalized in the interval [0.2-0.8]. In the input layer of the MLP there is a neuron for each of the parameters shown in Table 1 , except the Flux of $\mathrm{CO}_{2}$, which is the solution for the cases. The number of neurons selected for the hidden layer of the MLP is determined using the expression $2 n+1$, where $n$ is the number of neurons in the input layer. This value was defined 
following the criteria proposed by Kolmogorov [18]. Finally, the output layer of the MLP is composed of a neuron that represents the Flux of $\mathrm{CO}_{2}$ parameter shown in Table 1 . The training stage finishes when the cross validation, which uses $10 \%$ of the initial cases, provides an error rate that is lower than $\mu$. Once the MLP has been trained, the Jacobean Sensitivity Matrix is calculated and the Pondered Weigh Technique is applied.

Revise phase: Revision Simulated Equation (RSE): During the revision stage an equation $(F)$ is used to validate the proposed solution $\mathrm{p}^{*}$.

$$
F=k s o\left(p \mathrm{CO}_{2} \mathrm{SW}-\mathrm{pCO}_{2} \mathrm{AIR}\right)
$$

Where $\mathrm{F}$ is the flux of $\mathrm{CO} 2, k$ is the gas transfer velocity (3), so is the solubility verifying (4) and pCO2 is the partial pressure of $\mathrm{CO} 2$ (5).

$$
k=(-5,204 \text { Lat }+0,729 \text { Long }+2562,765) / 3600
$$

$S O=e^{\left(\frac{93,4517}{100 t k}-60,2409+23,3585 \log (100 t k)+s(0,023517-0,023656 \bullet 100 t k+0,0047036 \bullet 1002 t k)\right)}$

$$
p \mathrm{CO}_{2}=A+B L o n g+C L a t+D S S T+E Y e a r
$$

As can be seen in (6), $\mathrm{k}$ depends on Lat (Latitude), Long (Longitude). As can be seen in (7) so depends on $\mathrm{tk}=273,15+\mathrm{t}$. where $\mathrm{t}$ is the temperature and $\mathrm{s}$ is the salinity. Finally, in (8) it is possible to observe that pCO2 depends on the SST, which is the temperature of the marine surface or air as it corresponds to pCO2SW or pCO2AIR. The coefficients of the equation (8) depend on the month.

Retain phase: This phase begins once the prediction has been compared to the result provided using the mathematical model. If the case is considered valid (if the prediction differs by less than $10 \%$ ), the case is stored in the memory of cases. When this occurs, it is necessary to train the GCS network in order to include the new case in the structure of the memory of cases. In this way the new experience obtained processing the current case will be taken into consideration for the next prediction.

\section{Results and Conclusions}

The system described above was tested in the North Atlantic Ocean during 2003 and 2004. Although the system is not fully operational and the aim of the project is to construct a research prototype and not a commercial tool, the initial results have been very successful from a technical and scientific point of view. The construction of the distributed system was relatively simple, using previously developed CBR-BDI libraries [3, 4, 5, 3, 6]. facilitates the straight mapping between the agent definition and the CBR construction. The multiagent system automatically incorporated over 50,000 instances during the five months and eliminated $12 \%$ of the initial ones. 


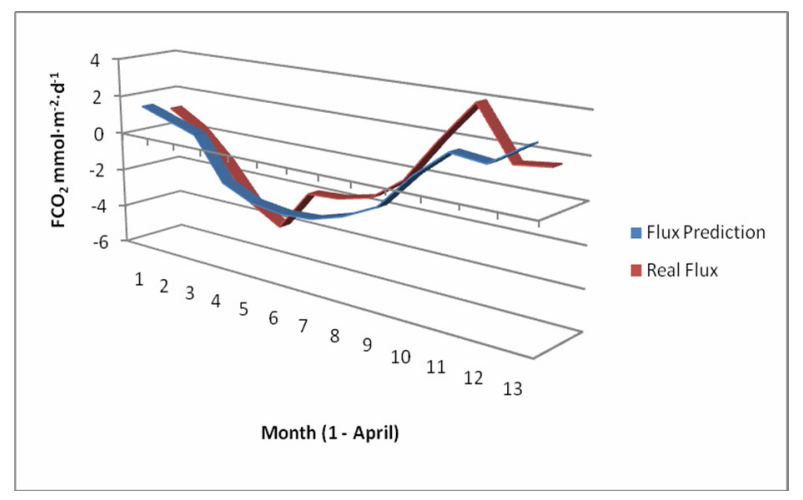

Fig. 2 Real CO2 flux and flux prediction

Figure 2 shows a comparison of the real data and the predictions provided by the multiagent system working with data from 2003-2004. As shown in Figure 2 the predictions provided by the multiagent system are accurate ( 9 of the 12 models were accepted as successful). The multiagent system makes predictions based on previous experiences, taking into account the similitude with past situations. Figure 2 shows how the precision of the prediction improves when the number of cases increases.

On the other hand, it is necessary to control the number of cases in the memory of cases in order to avoid an excessive growth of the cases available. To maintain the memory of cases, we used a strategy based on priorities, consisting of a pyramidal structure of efficiencies. The multiagent system facilitates the incorporation of new agents that use different modeling techniques and learning strategies, so our future work will focus on the incorporation of new agents with alternative techniques and the execution of additional experiments.

Acknowledgements. This work has been partially supported by the JCYL SA071A08 project.

\section{References}

1. Andrade, F., Novais, P., Machado, J., Neves, J.: Contracting agents: legal personality and representation. Artif. Intell. Law 15(4), 357-373 (2007)

2. Bratman, M.E., Israel, D., Pollack, M.E.: Plans and resource-bounded practical reasoning. Computational Intelligence 4, 349-355 (1988)

3. Bajo, J., Corchado, J.M., Alonso, V.: Sistema Multiagente para la Predicción del Intercambio de $\mathrm{CO}_{2}$ en el Océano Atlántico Norte. IEEE Latin America Transactions 6(6), 505-510 (2008)

4. Chaib-draa, B., Dignum, F.: Trends in Agent Communication Language. Computational Intelligence 18(2), 89-101 (2002)

5. Corchado, J.M., Laza, R.: Constructing Deliberative Agents with Case-based Reasoning Technology. International Journal of Intelligent Systems 18(12), 1227-1241 (2003) 
6. Corchado, J.M., Aiken, J., Corchado, E., Lefevre, N., Smyth, T.: Quantifying the Ocean's CO2 Budget with a CoHeL-IBR System. In: Funk, P., González Calero, P.A. (eds.) ECCBR 2004. LNCS (LNAI), vol. 3155, pp. 533-546. Springer, Heidelberg (2004)

7. Corchado, J.M., Bajo, J., Abraham, A.: GERAmI: Improving the delivery of health care. IEEE Intelligent Systems 23(2), 19-25 (2008)

8. Corchado, J.M., Aiken, J., Bajo, J.: A CBP Agent for Monitoring the CO2 Exchange Rate. Studies on Computational Intelligence, vol. 73, pp. 213-246 (2008)

9. Corchado, J.M., Bajo, J., de Paz, J.F., Rodríguez, S.: An execution time neural-CBR guidance system. NeuroComputing 72(13-15), 2743-2753, ISSN 0925-2312; Special Issue on Hybrid Intelligence in Neurocomputing (2009), doi:10.1016/j.neucom.2008.08.020

10. Corchado, J.M., Gonzalez-Bedia, M., De Paz, Y., Bajo, J., De Paz, J.F.: Replanning mechanism for deliberative agents in dynamic changing environments. Computational Intelligence 24(2), 77-107 (2008)

11. Dransfeld, S., Tatnall, A.R., Robinson, I.S., Mobley, C.D.: A comparison of Multilayer Perceptron and multilinear regression algorithms for the inversion of synthetic ocean colour spectra. Int. J. Remote Sens. 25(21), 4829-4834 (2004)

12. Ella Hassanien, A., Abraham, A., Grosan, C.: Spiking neural network and wavelets for hiding iris data in digital images. Soft Computing 13(4), 401-416 (2009)

13. Fritzke, B.: A growing neural gas network learns topologies. In: Tesauro, G., Touretzky, D.S., Leen, T.K. (eds.) Advances in Neural Information Processing Systems, Cambridge, MA, vol. 7, pp. 625-632 (1995)

14. Hruschka, E.R., Campello, R., Freitas, A., Carvalho, A.: A Survey of Evolutionary Algorithms for Clustering. IEEE Transactions on Systems, Man and Cybernetics - Part C: Applications and Reviews 39(2), 133-155 (2009)

15. Jayaputera, G.T., Zaslavsky, A.B., Loke, S.W.: Enabling run-time composition and support for heterogeneous pervasive multi-agent systems. Journal of Systems and Software 80(12), 2039-2062 (2007)

16. Lefevre, N., Aiken, J., Rutllant, J., Daneri, G., Lavender, S., Smyth, T.: Observations of pCO2 in the coastal upwelling off Chile: Sapatial and temporal extrapolation using satellite data. Journal of Geophysical Research 107 (2002)

17. Martinetz, T.: Competitive Hebbian learning rule forms perfectly topology preserving maps. In: Gielen, S., Kappen, B. (eds.) ICANN 1993: International Conference on Artificial Neural Networks, pp. 427-434. Springer, Amsterdam (1991)

18. Murtagh, F.: Multilayer perceptronsnext term for classification and regression. Neurocomputing 2(5-6), 183-197 (1991)

19. Saitou, N., Nie, M.: The neighbor-joining method "A new method for reconstructing phylogenetic trees”. Mol. Biol. 4, 406-425 (1987)

20. Sarmiento, J.L., Dender, M.: Carbon biogeochemistry and climate change. Photosynthesis Research 39, 209-234 (1994)

21. Schön, B., O’Hare, G.M.P., Duffy, B.R., Martin, A.N., Bradley, J.F.: Agent Assistance for 3D World Navigation. In: Panayiotopoulos, T., Gratch, J., Aylett, R.S., Ballin, D., Olivier, P., Rist, T. (eds.) IVA 2005. LNCS (LNAI), vol. 3661, p. 499. Springer, Heidelberg (2005)

22. Sheng, Y., Chen, G., Tan, K., Deshpande, U., Vance, B., Ho, Y., McDonald, C., Henderson, T., Kotz, D., Campbell, A., Wright, J.: MAP: A scalable monitoring system for dependable 802.11 wireless networks. IEEE Wireless Communications 15(5), 10-18 (2008)

23. Wooldridge, M., Jennings, N.R.: Intelligent Agents: Theory and Practice. The Knowledge Engineering Review 10(2), 115-152 (1995) 